\title{
Micro-CT in situ study of carbonate rock microstructural evolution for geologic $\mathrm{CO} 2$ storage
}

\author{
Zheng, Yi; Yang, Yan; Rogowska, M.; Gundlach, Carsten
}

\section{Published in:}

Proceedings of SPIE

Link to article, DOI:

$10.1117 / 12.2273877$

Publication date:

2017

Document Version

Publisher's PDF, also known as Version of record

Link back to DTU Orbit

Citation (APA):

Zheng, Y., Yang, Y., Rogowska, M., \& Gundlach, C. (2017). Micro-CT in situ study of carbonate rock microstructural evolution for geologic CO2 storage. In Proceedings of SPIE (Vol. 10391). [103910M ] SPIE International Society for Optical Engineering. Proceedings of SPIE - The International Society for Optical Engineering https://doi.org/10.1117/12.2273877

\section{General rights}

Copyright and moral rights for the publications made accessible in the public portal are retained by the authors and/or other copyright owners and it is a condition of accessing publications that users recognise and abide by the legal requirements associated with these rights.

- Users may download and print one copy of any publication from the public portal for the purpose of private study or research.

- You may not further distribute the material or use it for any profit-making activity or commercial gain

- You may freely distribute the URL identifying the publication in the public portal 


\section{Micro-CT in situ study of carbonate rock microstructural evolution for geologic $\mathrm{CO}<\mathrm{sub}>2</$ sub $>$ storage}

Y. Zheng, Y. Yang, M. Rogowska, C. Gundlach

Y. Zheng, Y. Yang, M. Rogowska, C. Gundlach, "Micro-CT in situ study of carbonate rock microstructural evolution for geologic $\mathrm{CO}<$ sub $>2</$ sub $>$ storage," Proc. SPIE 10391, Developments in X-Ray Tomography XI, 103910M (26 September 2017); doi: 10.1117/12.2273877

EDent: SPIE Optical Engineering + Applications, 2017, San Diego, California, United States 


\title{
Micro-CT in situ study of carbonate rock microstructural evolution for geologic $\mathrm{CO}_{2}$ storage
}

\author{
Y. Zheng*a, Y. Yang ${ }^{\mathrm{b}}$, M. Rogowska ${ }^{\mathrm{b}}$, C. Gundlach ${ }^{\mathrm{a}}$ \\ ${ }^{a}$ Dept. of Physics, Technical University of Denmark, DK-2500 Lyngby, Copenhagen, Denmark; ${ }^{\mathrm{b}}$ Nano- \\ Science Center, Dept. of Chemistry, University of Copenhagen, DK-2100 Copenhagen, Denmark
}

\begin{abstract}
To achieve the $2{ }^{\circ} \mathrm{C}$ target made in the 2016 Paris Agreement, it is essential to reduce the emission of $\mathrm{CO}_{2}$ into the atmosphere. Carbon Capture and Storage (CCS) has been given increasing importance over the last decade. One of the suggested methods for CCS is to inject $\mathrm{CO}_{2}$ into geologic settings such as the carbonate reservoirs in the North Sea. The final aim of our project is to find out how to control the evolution of petrophysical parameters during $\mathrm{CO}_{2}$ injection using an optimal combination of flow rate, injection pressure and chemical composition of the influent. The first step to achieve this is to find a suitable condition to create a stable 3D space in carbonate rock by injecting liquid to prepare space for the later $\mathrm{CO}_{2}$ injection. Micro-CT imaging is a non-destructive 3D method that can be used to study the property changes of carbonate rocks during and after $\mathrm{CO}_{2}$ injection. The advance in lab source based micro-CT has made it capable of in situ experiments. We used a commercial bench top micro-CT (Zeiss Versa XRM410) to study the microstructure changes of chalk during liquid injection. Flexible temporal CT resolution is essential in this study because that the time scales of coupled physical and chemical processes can be very different. The results validated the feasibility of using a bench top CT system with a pressure cell to monitor the mesoscale multiphase interactions in chalk.
\end{abstract}

Keywords: Micro-CT, porosity, in situ, oil reservoir, 4D computed tomography

\section{INTRODUCTION}

In order to captures the changing structure in an in situ experiment, the scanning time of one micro-CT scan as well as the time between two consequent scans need to be sufficiently short. If the scanning time is too long compared to the structure changing time, not only the time stamp of the scan is incorrect but will result in motion artifact which create blurring in the reconstructed images. If the time between two scans is too long, then the events between two scans cannot be recorded.

A reduction in the number of projections will result in a reduction of scanning time given the same exposure time. However, a reduction in the number of projections often means a reduction in data quality; this is especially true for analytic reconstruction methods (e.g. the filtered back-projection algorithm).

An alternative is to use an iterative method. The advantages of the iterative method compared with the analytic method are the improved insensitivity to noise and the possibility to reconstruct the object with fewer projections using prior knowledge. Although first introduced in the 1970s ${ }^{1}$, the application of iterative reconstruction has been limited due to the high demand of computer memory and speed. However, the recent advance of computing power has made the iterative reconstruction methods more accessible.

*yizhe@fysik.dtu.dk

Developments in X-Ray Tomography XI, edited by Bert Müller, Ge Wang, Proc. of SPIE Vol. 10391, 103910M · C 2017 SPIE · CCC code: 0277-786X/17/\$18 · doi: 10.1117/12.2273877 
The ASTRA Toolbox (All Scales Tomographic Reconstruction Antwerp) is an efficient and flexible open source tools for tomography reconstruction and simulation ${ }^{2,3}$. The SIRT (Simultaneous Iterative Reconstruction Technique) algorithm implemented in the ASTRA Toolbox is an algebraic technique, which has been recommended for limited projections by several authors ${ }^{4,5}$. One limitation of the iterative methods on $3 \mathrm{D}$ reconstruction is the slow processing speed. The ASTRA Toolbox was mostly written in $\mathrm{C}++$ with its core computations offloaded to a GPU card to enable fast computing speed.

\section{MATERIALS AND METHODS}

\subsection{Samples and experimental setup}

A cylindrical chalk sample $(\varnothing \times \mathrm{L}=0.9 \times 2 \mathrm{~mm})$ obtained from outcrop Maastrichtian chalk collected near Aalborg, Denmark (Rørdal Quarry) was used in our experiment. The sample was loaded in a mini Hassler core holder (Figure 1). Double distilled water was forced through the sample with a constant flowrate of $0.01 \mathrm{ml} / \mathrm{min}$. The experiment took place in a ZEISS Versa XRM410 micro-CT with a 4X objective lens and 120k peak voltage. In total, 18 consecutive micro-CT was obtained over a period of 46 hours. Each scan took around 2.5 hours and consisted of 1601 projections covering $360^{\circ}$, with a voxel size of $3.2 \mu \mathrm{m}$.

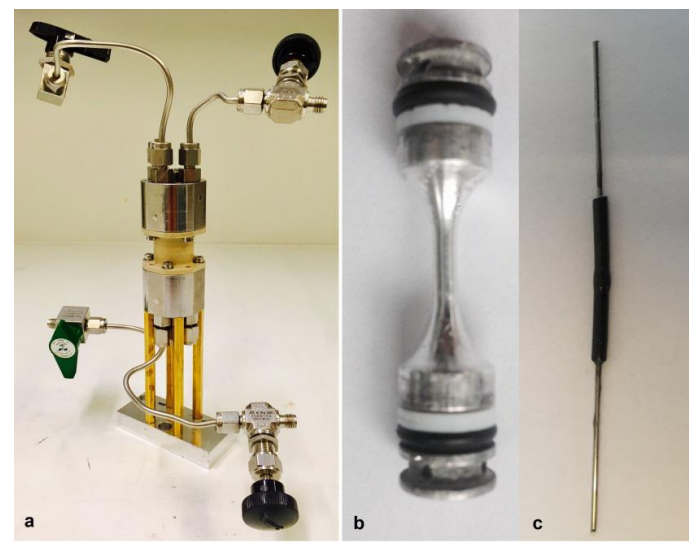

Figure 1. Photographs of a high pressure cell (a) and an aluminium holder (b) for a sample (c).

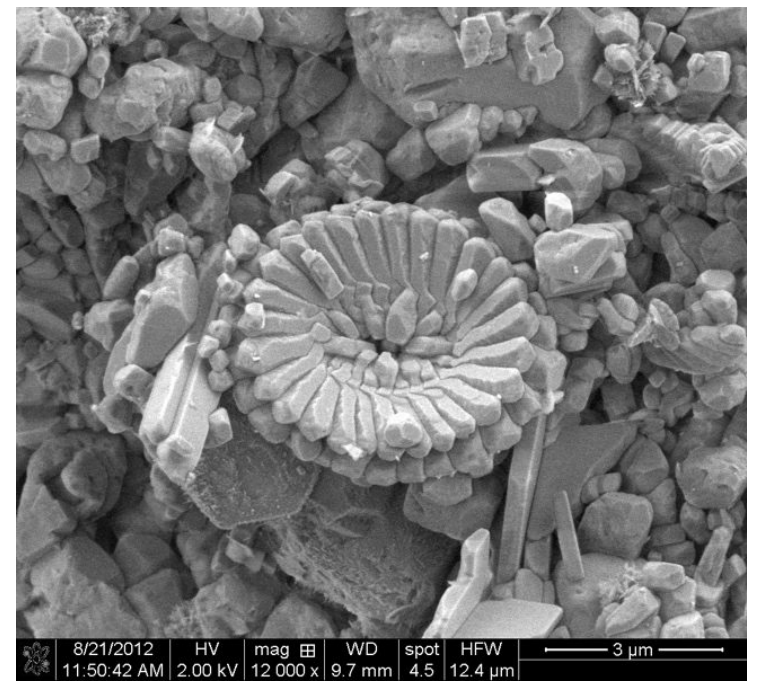

(a)

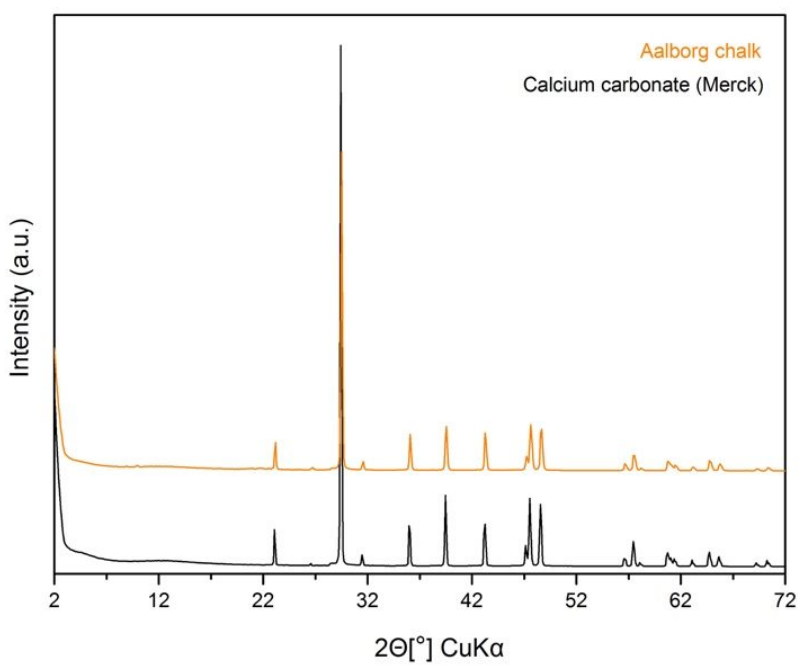

(b)

Figure 2. SEM image (a) and XRD patterns (b) of Aalborg chalk sample. 


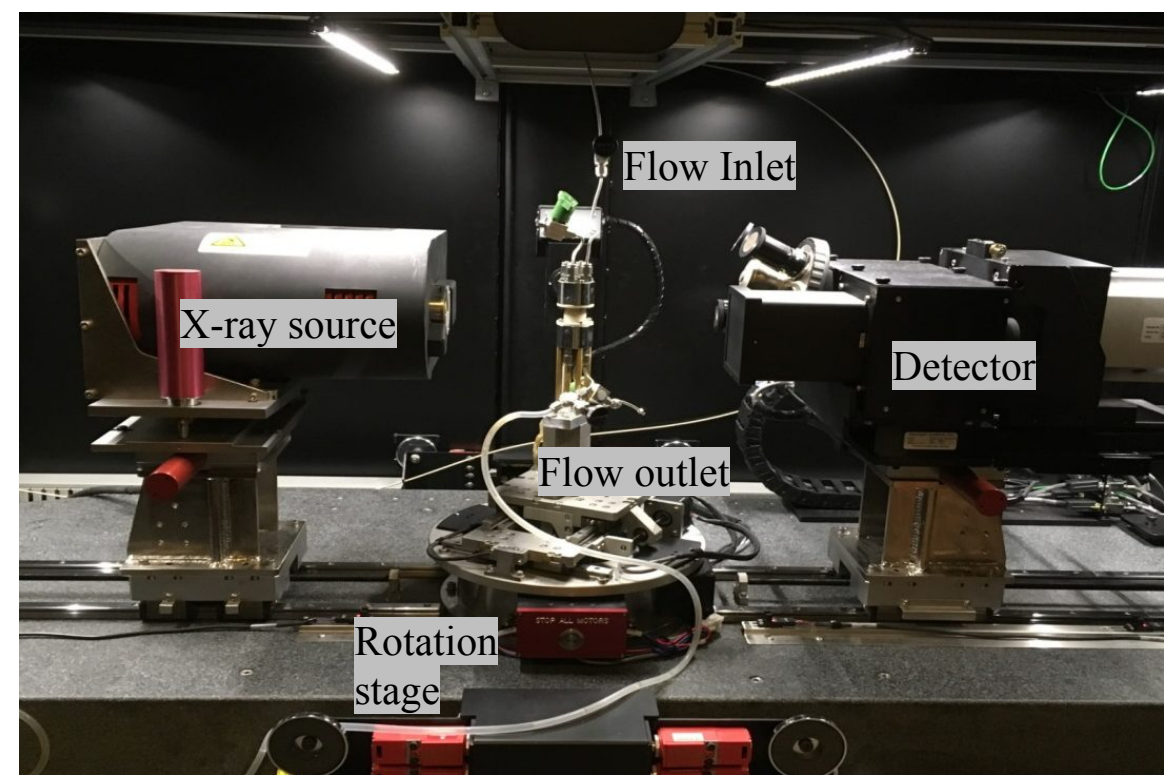

Figure 3.Micro-CT in situ experimental setup

\subsection{Reconstruction method}

By using the ASTRA Toolbox, we were able to investigate the image quality when using fewer projections. We resample the projection data evenly through $360^{\circ}$ using (a) 1601 (b) 801 (c) 401 (d) 201 projections of the first micro-CT dataset.

Both a short scanning time and the gap between two scans are important for in situ experiments. In this paper, we used the method described in Figure 4 to shorten the gap between two scans. Figure 4 illustrates the method, where the 1601 projections covering $360^{\circ}$ are resampled to 8 datasets and each data subset covers $180^{\circ}$ but starting at different rotation angles. In our experiment, the first data subset is the first 827 projections, and covers $180^{\circ}$ plus the cone beam angle, while the second data subset starts from the $100_{\text {th }}$ projection, corresponding to $\sim 45^{\circ}$. In this way, the time difference between the two subsets is only $1 / 8$ of the acquisition time.

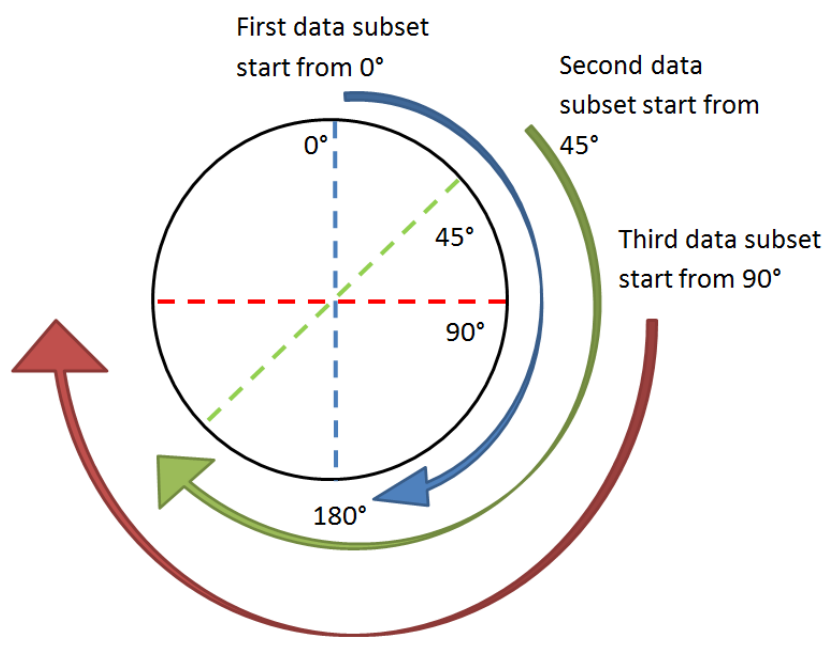

Figure 4. Schematic showing re-sampling one micro-CT dataset of $360^{\circ}$ to different sub dataset: in this way, the time difference between two sub dataset is the acquisition time of only $45^{\circ}$. In our case, this is about 20 minutes. 


\section{RESULTS}

Gray value of the micro-CT reconstructed images is an arbitrary unit. This gray value can change due to the energy spectrum of the X-ray source, the sample thickness, reconstruction method and reconstruction setting, etc.. During the experiment, the range of gray value in each in situ micro-CT scan slightly differs from one to another. A registration of the gray value using its histogram was used to normalize the reconstruction images in different time steps. As can be shown in Figure 5, it is visible that there are five peaks in the histogram of the reconstruction images. The histogram given in Figure 5 is the histogram of 291 slices, which consists of 293002080 voxels. The value 0 was not taken into account in the histogram. This is because the 0 value was given by the reconstruction software to a voxel that is outside the reconstructed field of view. The gray value of air, water, and the metal tube should be relatively stable from one scan to another. Therefore, the peak position of these three components can be used for normalization of the gray value. The density of these three materials are also known, this makes it possible to register the gray value to absolute density.

Figure 6 shows how the density changes of the sample through 46 hours. There is a reduction of overall density and an expansion of void through the process. Figure 7 shows how the void of the sample expanded over time. Although the commercial micro-CT was able to record the carbonate rock microstructural evolution, the acquisition is relatively slow ( 2.5 hours per scan). This means that the reconstructed CT images can only show the average structure of the carbonate rock within this 2.5 hours window. In order to increase the time resolution, the method described in Section 2.2 was used to reduce the time difference between two scans to $\sim 20$ minutes.

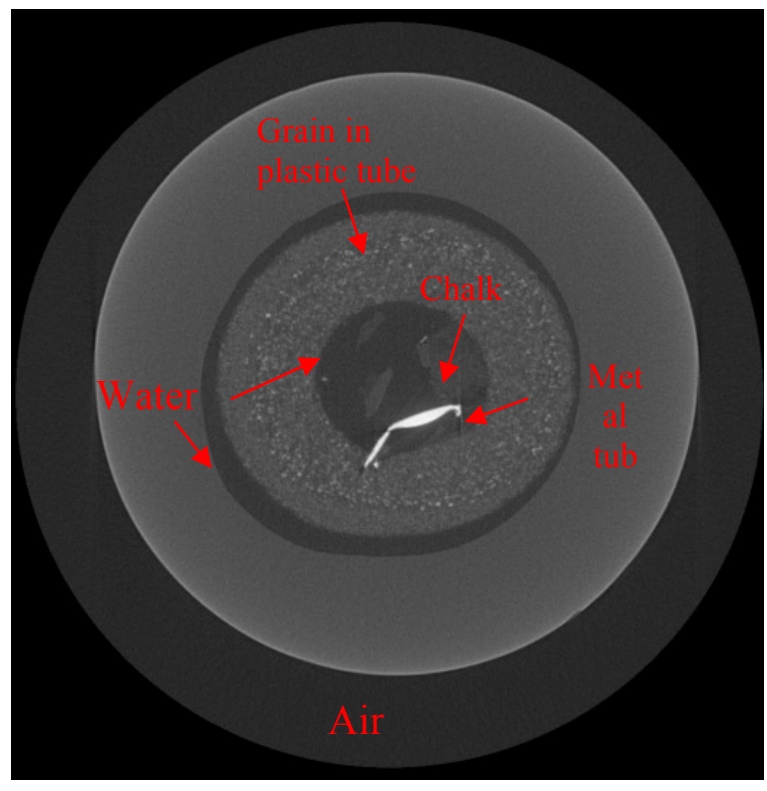

(a)

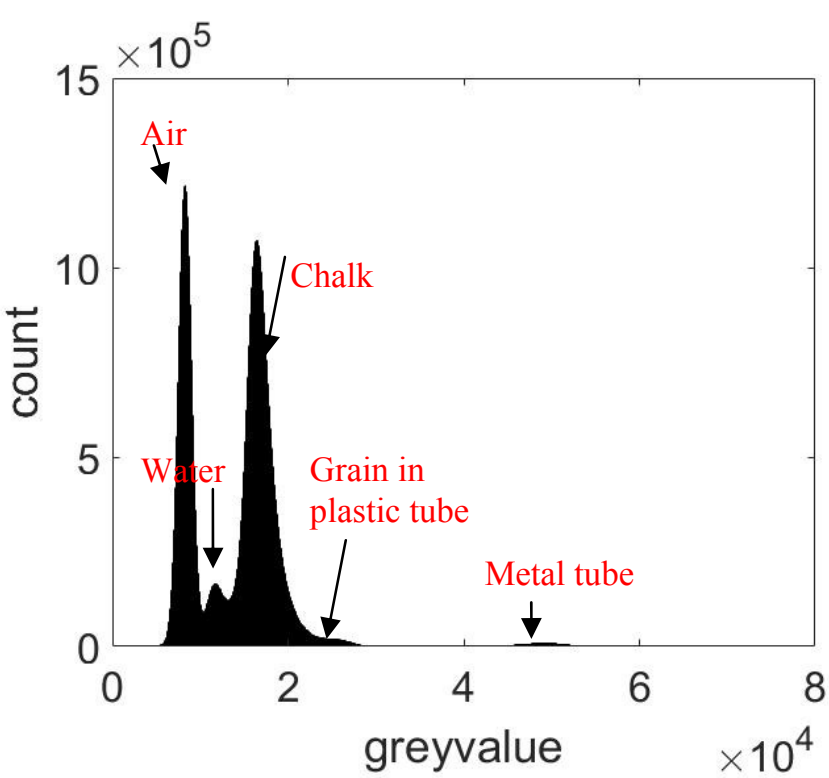

(b)

Figure 5. Reconstructed slice (a) of the sample and histogram (b) indicating different components in the images 


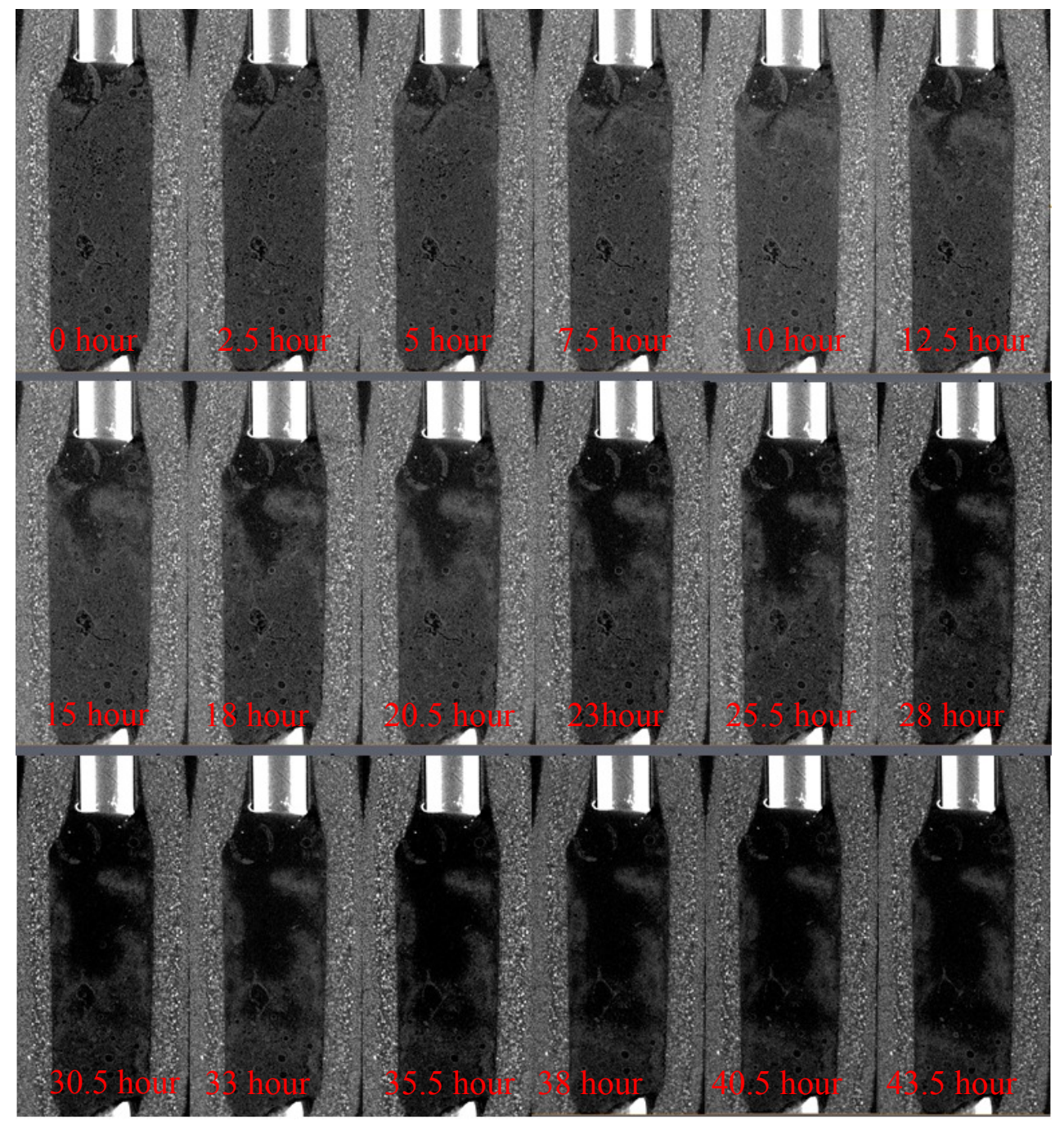

Figure 6. Reconstructed slices of the center axis of the sample through 46 hours, each scan takes 2.5 hours

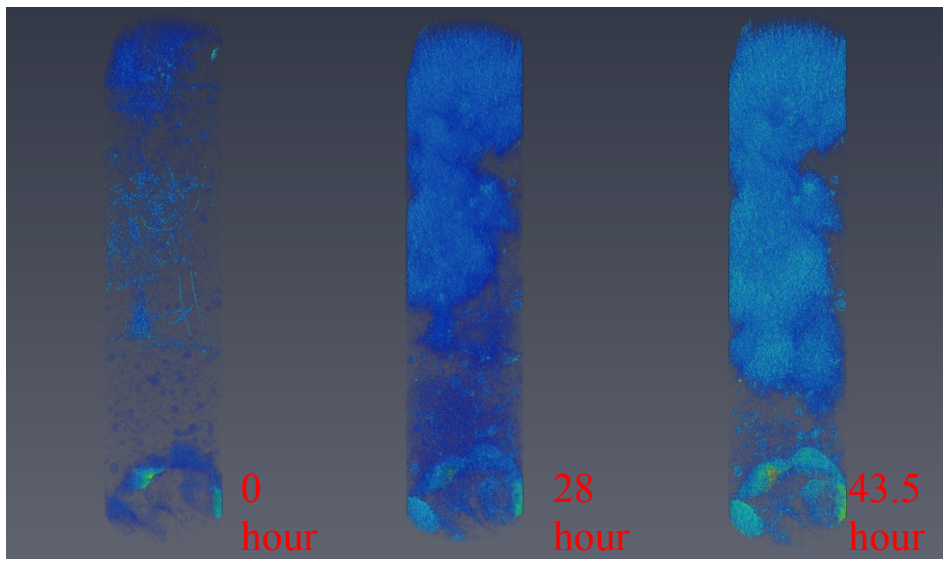

Figure 7. Void expansion shown in 3D micro-CT images: the volume of the chalk sample was removed so that the figure is only showing the void within the sample. 

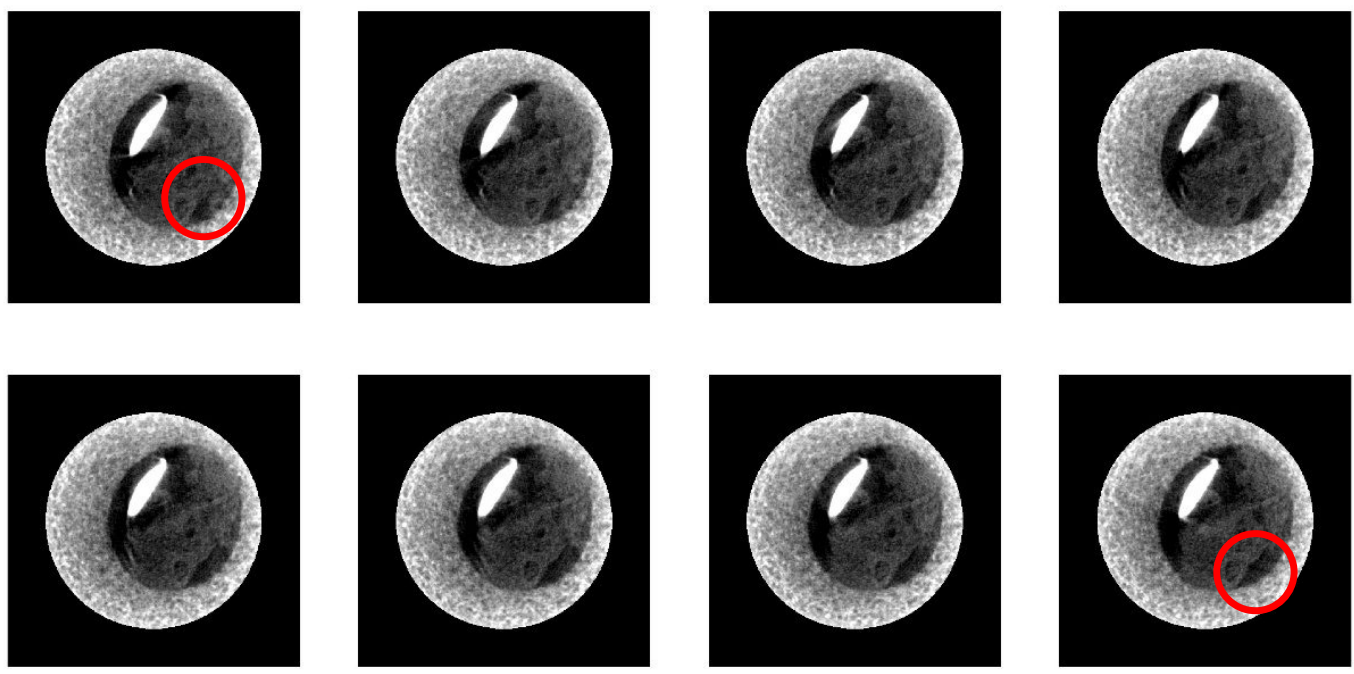

Figure 8. Reconstruction slices after resampling of the original projection data: from left to the right and from top to bottom, each slice represent has a time difference of $\sim 20$ minutes. Notice the difference between different sub dataset within area of the red circles.

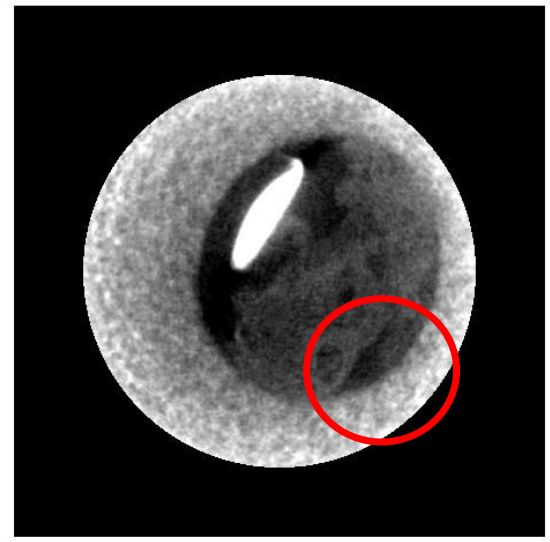

Figure 9. Original reconstructed slice using the full dataset (1601 projections) 


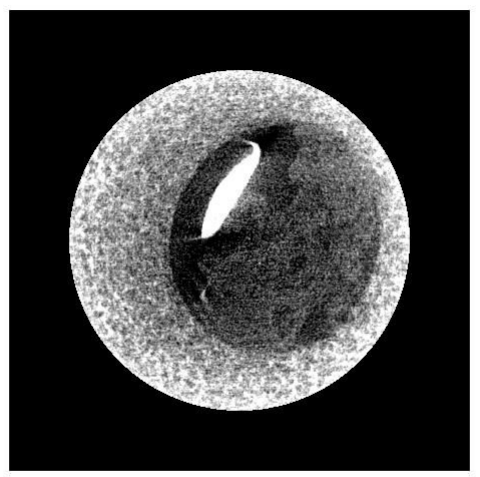

(a)

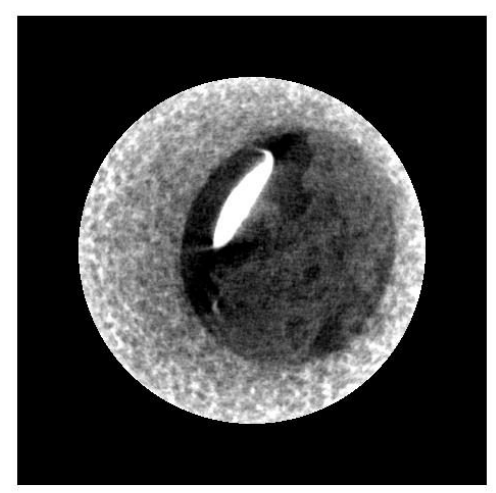

(b)

Figure 10.Comparing reconstruction slice using Kemp (FDK) cone beam reconstruction and (Simultaneous Iterative Reconstruction Technique) SIRT methods when using $180^{\circ}$ plus cone beam degree.

By re-sampling one micro-CT acquisition (1601 projections) to 8 different subsets with a time difference of 20 minutes, information correlated to narrower time window is presented. As can be seen in Figure 8, a small void was growing from the first image to the last, which cannot be seen when using the whole dataset (see Figure 9). The time difference between two data subsets in Figure 8 is $\sim 20$ minutes.

Kemp (FDK) cone beam reconstruction is an analytic reconstruction method based on filter back projection for cone beam geometry. It requires a large number of projections to reconstruct the $3 \mathrm{D}$ volume. A reduction in the number of projections deteriorates image quality as shown in Figure 10 (a). When using traditional FDK methods ${ }^{6}$ of fewer projections, the image was disrupted by noise. In contrast, when using SIRT, the image quality in Figure 10 (b) is comparable to that which uses the full dataset (Figure 9, $360^{\circ}$ ).

By reconstructing the micro-CT data using fewer projections, the acquisition time of one micro-CT scan can be dramatically reduced. Figure 11 shows the reconstructed slices when using few projections. Our results show that the features of the sample are still visible by using just $25 \%$ of the projections, this make up an acquisition time of 40 minutes.

By using the iterative reconstruction method in Astra, we are able to reduce the acquisition time of each micro-CT scan from 2.5 hours to 40 minutes, almost quadrupling the temporal resolution. Another improvement is that by resampling the data from different starting point, the time difference between two subsequent micro-CT dataset is no longer restricted by the acquisition time. 

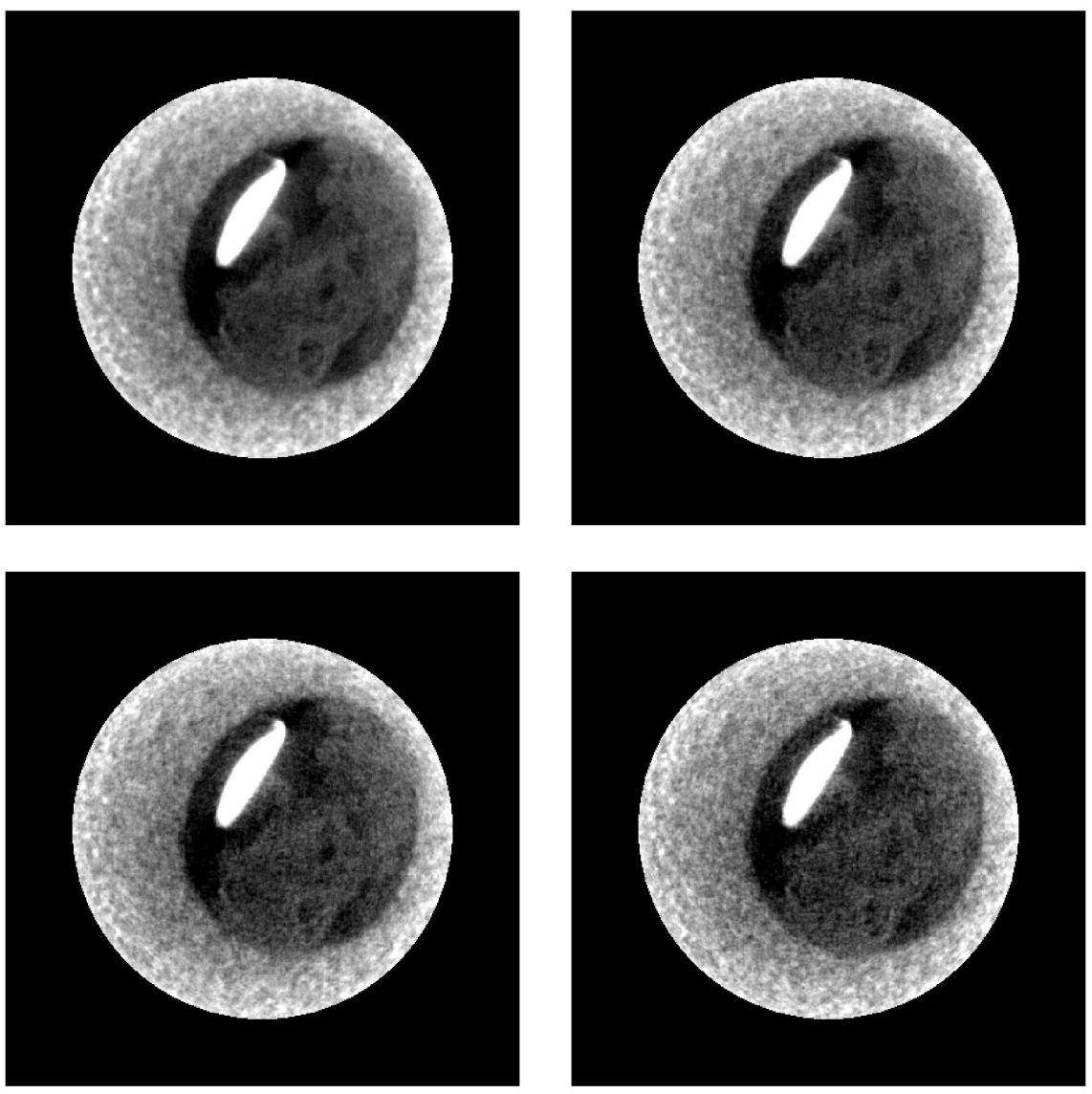

Figure 11. Comparing reconstructed slices with reducing projection number over $360^{\circ} \mathrm{C}$ (a) 1601 (b) 801 (c) 401 (d) 201

\section{CONCLUSIONS AND FUTURE WORK}

Our results show that the ASTRA is a powerful micro-CT reconstruction toolbox for real and continuous datasets. By resampling time steps, the time gap between two consecutive micro-CT scans is no longer limited by the acquisition time. By using iterative method, it is possible to reduce the acquisition time while maintaining image quality. The next step will be to investigate the influence of the new method in our analysis results (such as porosity changes) to establish a protocol for in situ studies. 


\section{REFERENCES}

[1] Houndfiedl, G. N. "Computerized transverse axial scanning (tomography). 1. Description of system". Br. J. Radiol. 46, 1016-1022 (1973).

[2] Van Aarle, W. et al. "The ASTRA Toolbox: A platform for advanced algorithm development in electron tomography". Ultramicroscopy 157, 35-47 (2015).

[3] Van Aarle, W. et al. "Fast and flexible X-ray tomography using the ASTRA toolbox". Opt. Express 24, 25129-25147 (2016).

[4] Verhoeven, D." Limited-data computed tomography algorithms for the physical sciences." Appl. Opt. 32, 3736-3754 (1993).

[5] Subbarao, P. M. V, Munshi, P. \& Muralidhar, K. "Performance of iterative tomographic algorithms applied to non-destructive evaluation with limited data". NDT E Int. 30, 359-370 (1997).

[6] Feldkamp, L. A., Davis, L. C. \& Kress, J. W. "Practical cone-beam algorithm". J. Opt. Soc. Am. A-OPTICS IMAGE Sci. Vis. 1, 612-619 (1984). 